\title{
Structural Studies of Coronavirus Fusion Proteins
}

Alexandra C. Walls ${ }^{1}$, M. Alejandra Tortorici ${ }^{1,2}$, Xiaoli Xiong ${ }^{1}$, Joost Snijder ${ }^{1}$, Brandon Frenz ${ }^{1}$, BerendJan Bosch $^{3}$, Frank DiMaio ${ }^{1}$, Davide Corti ${ }^{4}$, Félix A. Rey ${ }^{2}$ and David Veesler ${ }^{1 *}$

1. Department of Biochemistry, University of Washington, Seattle, Washington 98195, USA.

2. Institut Pasteur, Unité de Virologie Structurale, Paris 75015, France.

3. Virology Division, Department of Infectious Diseases and Immunology, Faculty of Veterinary Medicine, Utrecht University, 3584 CL Utrecht, The Netherlands.

4. Humabs Biomed SA, Vir Biotechnology, 6500 Bellinzona, Switzerland.

* Corresponding author: dveesler@uw.edu

Coronaviruses are zoonotic viruses responsible for mild respiratory tract infections and fatal pneumonia in humans worldwide (e.g. SARS: severe acute respiratory syndrome or MERS: Middle-East respiratory syndrome)[1]. The spike glycoprotein is responsible for coronavirus entry into target cells, carrying both receptor binding and membrane fusion functions, and is the main target of neutralizing antibodies upon infection[2]. The spike protein had resisted structural characterization for more than a decade using traditional approaches and this knowledge gap hindered rational development of inhibitors against these viruses. As a result, no vaccines or therapeutics are available against any of the six human-infecting coronaviruses.

We obtained the first atomic-level description of a coronavirus spike glycoprotein in the prefusion and postfusion conformations using cryo-electron microscopy (cryoEM)[3, 4]. This work provided snapshots of the start and end points of the membrane fusion reaction which is mediated by this spring-loaded nanomachine to promote viral entry into host cells. Moreover, these findings indicated potential strategies to neutralize these pathogens. We also integrated cryoEM and glycoproteomics to characterize the spike glycoproteins of human and animal pathogenic coronaviruses (SARS-CoV, MERS-CoV, HCoV-NL63 and PDCoV)[5-7]. We revealed the presence of a composite glycan shield, comprising up to one hundred N-linked oligosaccharides, obstructing the surface of coronavirus spike glycoproteins. Our work suggested coronaviruses use molecular trickery, based on epitope masking with glycans and activating conformational changes, to evade the immune system of infected hosts.

We recently studied the mechanisms of SARS-CoV and MERS-CoV neutralization by two potent human neutralizing antibodies isolated from survivors[6]. Although we found both antibodies blocked receptor-interaction, the anti-SARS-CoV antibody also acted by functionally mimicking receptorattachment and promoting spike fusogenic conformational rearrangements through a ratcheting mechanism. This finding is an unprecedented example of functional mimicry, whereby an antibody activates membrane fusion by recapitulating the action of the receptor. Furthermore, these findings elucidated the unique nature of coronavirus membrane fusion activation. I will be presenting the approaches we developed to prepare coronavirus spike protein samples for high-resolution cryoEM studies, such as capturing a transient complex between the SARS-CoV spike glycoprotein and a 
neutralizing antibody (Figure 1). Finally, I will be discussing methods that allowed us to overcome specimen orientation anisotropy in vitrous ice.

\section{References}

1. Su S, et al. (2016) Epidemiology, Genetic Recombination, and Pathogenesis of Coronaviruses. Trends Microbiol 24(6):490-502.

2. Bosch BJ, van der Zee R, de Haan CA, \& Rottier PJ (2003) The coronavirus spike protein is a class I virus fusion protein: structural and functional characterization of the fusion core complex. Journal of virology 77(16):8801-8811.

3. Walls AC, et al. (2016) Cryo-electron microscopy structure of a coronavirus spike glycoprotein trimer. Nature 531(7592):114-117.

4. Walls AC, et al. (2017) Tectonic conformational changes of a coronavirus spike glycoprotein promote membrane fusion. Proceedings of the National Academy of Sciences 114(42):11157-11162.

5. Walls AC, et al. (2016) Glycan shield and epitope masking of a coronavirus spike protein observed by cryo-electron microscopy. Nature structural \& molecular biology 23(10):899-905.

6. Walls AC, et al. (2019) Unexpected Receptor Functional Mimicry Elucidates Activation of Coronavirus Fusion. Cell 176(5):1026-1039 e1015.

7. Xiong X, et al. (2017) Glycan shield and fusion activation of a deltacoronavirus spike glycoprotein fine-tuned for enteric infections. Journal of virology.

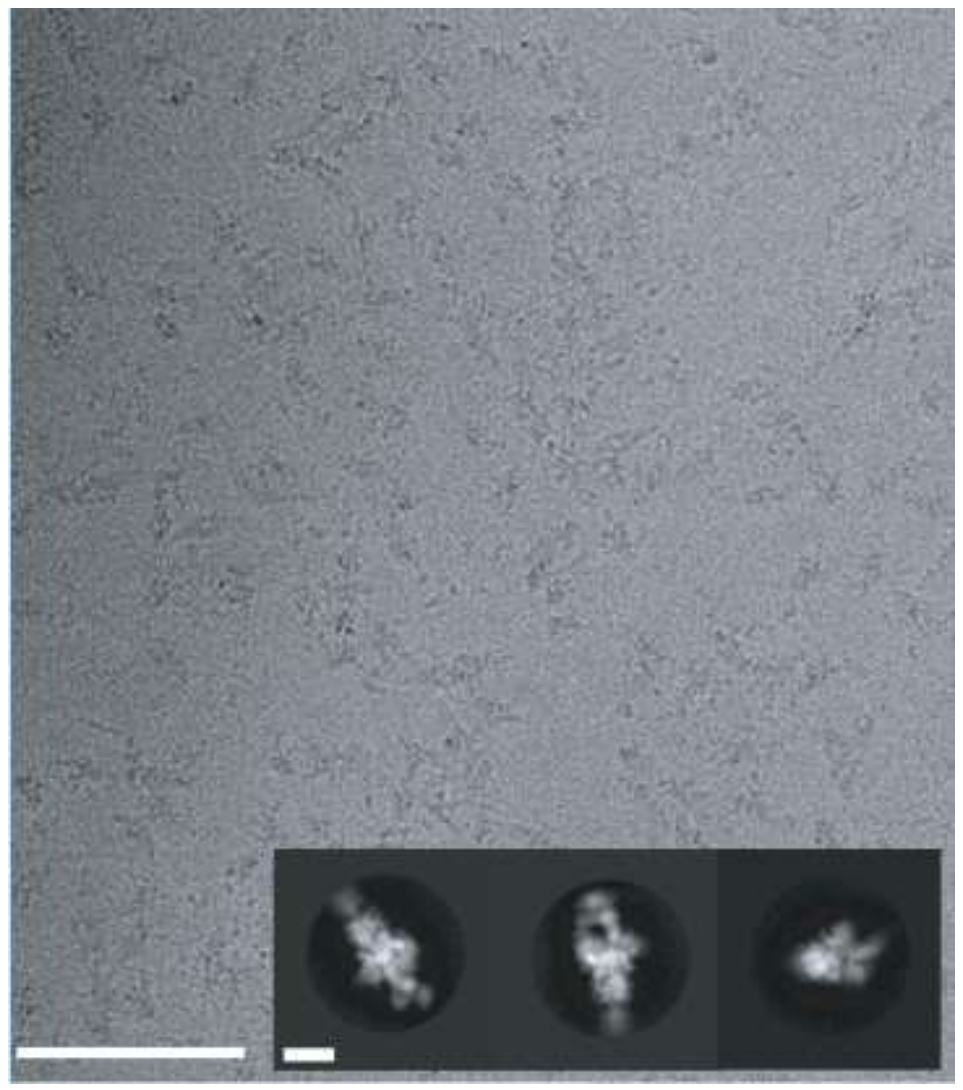

Figure 1. CryoEM characterization of the SARS-CoV S glycoprotein bound to the S230 Fab Fragment. Representative micrograph (scale bar: $100 \mathrm{~nm}$ ) and reference-free 2D class averages (scale bar: $100 \AA$ ). 